\title{
An inequality for $q$-integral and its applications
}

Mingjin Wang ${ }^{*}$

\section{"Correspondence:} wang197913@126.com Department of Applied Mathematics, Changzhou University, Changzhou, Jiangsu 213164, P.R. China

\section{Abstract}

In this paper, we use the $q$-binomial theorem to establish an inequality for the $q$-integral. As applications of the inequality, we give some sufficient conditions for convergence of the $q$-integral.

MSC: Primary 26D15; secondary 33D15

Keywords: inequality; $q_{\text {-integral; the basic hypergeometric function }}^{r+1} \phi_{r ;}$; the q-binomial theorem; convergence

\section{Introduction and main result}

$q$-Series, which are also called basic hypergeometric series, play a very important role in many fields, such as affine root systems, Lie algebras and groups, number theory, orthogonal polynomials, physics, etc. The inequality technique is one of the useful tools in the study of special functions. There are many papers about the inequalities and $q$-integral; see [1-10]. Convergence is the key problem of a $q$-series. In order to give some new methods for convergence of a $q$-series, we derive an inequality for the $q$-integral with the basic hypergeometric series ${ }_{r+1} \phi_{r}$. Some applications of the inequality are also given. The main result of this paper is the following inequality.

Theorem 1.1 Suppose $a_{i}, b_{i}, t$ be any real numbers such that $|t|<\left(\prod_{i=1}^{r+1} M_{i}\right)^{-1}$ and $b_{i}<1$ with $i=1,2, \ldots, r$. Then we have

$$
\left|\int_{0}^{t}{ }_{r+1} \phi_{r}\left(\begin{array}{c}
a_{1}, a_{2}, \ldots, a_{r+1} \\
b_{1}, b_{2}, \ldots, b_{r}
\end{array} q, z\right) d_{q} z\right| \leq \frac{|t|}{\left(1-|t| \prod_{i=1}^{r+1} M_{i}\right)\left(q^{2} ; q\right)_{\infty}}
$$

where $b_{r+1}=0, M_{i}=\max \left\{1, \frac{\left|1-a_{i}\right|}{1-b_{i}}\right\}$ for $i=1,2, \ldots, r+1$.

Before we present the proof of the theorem, we recall some definitions, notation, and known results which will be used in this paper. Throughout the whole paper, it is supposed that $0<q<1$. The $q$-shifted factorials are defined as

$$
(a ; q)_{0}=1, \quad(a ; q)_{n}=\prod_{k=0}^{n-1}\left(1-a q^{k}\right), \quad(a ; q)_{\infty}=\prod_{k=0}^{\infty}\left(1-a q^{k}\right) .
$$


We also adopt the following compact notation for a multiple $q$-shifted factorial:

$$
\left(a_{1}, a_{2}, \ldots, a_{m} ; q\right)_{n}=\left(a_{1} ; q\right)_{n}\left(a_{2} ; q\right)_{n} \cdots\left(a_{m} ; q\right)_{n}
$$

where $n$ is an integer or $\infty$.

The $q$-binomial theorem is $[11,12]$

$$
\sum_{k=0}^{\infty} \frac{(a ; q)_{k} z^{k}}{(q ; q)_{k}}=\frac{(a z ; q)_{\infty}}{(z ; q)_{\infty}}, \quad|z|<1
$$

Heine introduced the ${ }_{r+1} \phi_{r}$ basic hypergeometric series, which is defined by [11, 12]

$$
{ }_{r+1} \phi_{r}\left(\begin{array}{c}
a_{1}, a_{2}, \ldots, a_{r+1} \\
b_{1}, b_{2}, \ldots, b_{r}
\end{array} q, z\right)=\sum_{n=0}^{\infty} \frac{\left(a_{1}, a_{2}, \ldots, a_{r+1} ; q\right)_{n} z^{n}}{\left(q, b_{1}, b_{2}, \ldots, b_{r} ; q\right)_{n}} .
$$

Jackson defined the $q$-integral by [13]

$$
\int_{0}^{d} f(t) d_{q} t=d(1-q) \sum_{n=0}^{\infty} f\left(d q^{n}\right) q^{n}
$$

and

$$
\int_{c}^{d} f(t) d_{q} t=\int_{0}^{d} f(t) d_{q} t-\int_{0}^{c} f(t) d_{q} t
$$

In [14], the author gives the following inequality.

Theorem 1.2 Suppose $a_{i}, b_{i}$ and $z$ be any real numbers such that $|z|<\left(\prod_{i=1}^{r+1} M_{i}\right)^{-1}, b_{i}<1$ with $i=1,2, \ldots, r$. Then we have

$$
\left|r+1 \phi_{r}\left(\begin{array}{c}
a_{1}, a_{2}, \ldots, a_{r+1} \\
b_{1}, b_{2}, \ldots, b_{r}
\end{array} q, z\right)\right| \leq \frac{1}{\left(|z| \prod_{i=1}^{r+1} M_{i} ; q\right)_{\infty}},
$$

where $b_{r+1}=0, M_{i}=\max \left\{1, \frac{\left|1-a_{i}\right|}{1-b_{i}}\right\}$ for $i=1,2, \ldots, r+1$.

As an application of (1.8), the author give the following sufficient condition for convergence of $q$-series [14].

Theorem 1.3 Suppose $a_{i}, b_{i}$, $t$ be any real numbers such that $|t|<1$ and $b_{i}<1$ with $i=$ $1,2, \ldots, r$. Let $\left\{c_{n}\right\}$ be any number series. If

$$
\lim _{n \rightarrow \infty}\left|\frac{c_{n+1}}{c_{n}}\right|=p<1
$$

then the q-series

$$
\sum_{n=0}^{\infty} c_{n} \cdot{ }_{r+1} \phi_{r}\left(\begin{array}{c}
a_{1}, a_{2}, \ldots, a_{r+1} \\
b_{1}, b_{2}, \ldots, b_{r}
\end{array} ;, t q^{n}\right)
$$

converges absolutely. 


\section{Proof of theorem}

In this section, we use Theorems 1.2 and 1.3 to prove Theorem 1.1.

Proof First we point out that, under the conditions of Theorem 1.1, the $q$-integral

$$
\int_{0}^{t}{ }_{r+1} \phi_{r}\left(\begin{array}{c}
a_{1}, a_{2}, \ldots, a_{r+1} \\
b_{1}, b_{2}, \ldots, b_{r}
\end{array} ;, z\right) d_{q} z
$$

converges absolutely.

In fact, by the definition of $q$-integral (1.6), we get

$$
\begin{aligned}
& \int_{0}^{t}{ }_{r+1} \phi_{r}\left(\begin{array}{c}
\left.a_{1}, a_{2}, \ldots, a_{r+1} ; q, z\right) d_{q} z \\
b_{1}, b_{2}, \ldots, b_{r}
\end{array}\right. \\
& \quad=t(1-q) \sum_{n=0}^{\infty} q^{n}{ }_{r+1} \phi_{r}\left(\begin{array}{c}
a_{1}, a_{2}, \ldots, a_{r+1} ; q, t q^{n} \\
b_{1}, b_{2}, \ldots, b_{r}
\end{array}\right) .
\end{aligned}
$$

Using Theorem 1.3 and noticing

$$
\lim _{n \rightarrow \infty} \frac{q^{n+1}}{q^{n}}=q<1,
$$

we see that the $q$-integral (2.1) converges absolutely.

Letting $z=t q^{n}$ in (1.8) gives

$$
\left|{ }_{r+1} \phi_{r}\left(\begin{array}{c}
a_{1}, a_{2}, \ldots, a_{r+1} ; q, t q^{n} \\
b_{1}, b_{2}, \ldots, b_{r}
\end{array}\right)\right| \leq \frac{1}{\left(|t| q^{n} \prod_{i=1}^{r+1} M_{i} ; q\right)_{\infty}},
$$

where $b_{r+1}=0, M_{i}=\max \left\{1, \frac{\left|1-a_{i}\right|}{1-b_{i}}\right\}$ for $i=1,2, \ldots, r+1$.

Using the definition of $q$-integral (1.6) again one gets

$$
\begin{aligned}
& \mid \int_{0}^{t}{ }_{r+1} \phi_{r}\left(\begin{array}{c}
\left.a_{1}, a_{2}, \ldots, a_{r+1} ; q, z\right) d_{q} z \mid \\
b_{1}, b_{2}, \ldots, b_{r}
\end{array} \mid\right. \\
& \quad \leq\left.|t|(1-q) \sum_{n=0}^{\infty} q^{n}\right|_{r+1} \phi_{r}\left(\begin{array}{c}
a_{1}, a_{2}, \ldots, a_{r+1} ; q, t q^{n} \\
b_{1}, b_{2}, \ldots, b_{r}
\end{array}\right) \mid \\
& \quad \leq|t|(1-q) \sum_{n=0}^{\infty} \frac{q^{n}}{\left(|t| q^{n} \prod_{i=1}^{r+1} M_{i} ; q\right)_{\infty}} \\
& \quad=\frac{|t|(1-q)}{\left(|t| \prod_{i=1}^{r+1} M_{i} ; q\right)_{\infty}} \sum_{n=0}^{\infty}\left(|t| \prod_{i=1}^{r+1} M_{i} ; q\right)_{n} q^{n} \\
& \quad \leq \frac{|t|(1-q)}{\left(|t| \prod_{i=1}^{r+1} M_{i} ; q\right)_{\infty}} \sum_{n=0}^{\infty} \frac{\left(|t| \prod_{i=1}^{r+1} M_{i} ; q\right)_{n} q^{n}}{(q ; q)_{n}} .
\end{aligned}
$$

Employing the $q$-binomial theorem (1.4) gives

$$
\sum_{n=0}^{\infty} \frac{\left(|t| \prod_{i=1}^{r+1} M_{i} ; q\right)_{n} q^{n}}{(q ; q)_{n}}=\frac{\left(q|t| \prod_{i=1}^{r+1} M_{i} ; q\right)_{\infty}}{(q ; q)_{\infty}} .
$$

Substituting (2.5) into (2.4), we get (1.1). 
Corollary 2.1 Suppose $a_{i}, b_{i}, c, d$ be any real numbers such that $|c|<\left(\prod_{i=1}^{r+1} M_{i}\right)^{-1},|d|<$ $\left(\prod_{i=1}^{r+1} M_{i}\right)^{-1}$ and $b_{i}<1$ with $i=1,2, \ldots, r$. Then we have

$$
\begin{aligned}
& \mid \int_{c}^{d}{ }_{r+1} \phi_{r}\left(\begin{array}{c}
\left.a_{1}, a_{2}, \ldots, a_{r+1} ; q, z\right) d_{q} z \\
b_{1}, b_{2}, \ldots, b_{r}
\end{array} \mid\right. \\
& \quad \leq \frac{|d|+|c|-2|d c| \prod_{i=1}^{r+1} M_{i}}{\left(1-|d| \prod_{i=1}^{r+1} M_{i}\right)\left(1-|c| \prod_{i=1}^{r+1} M_{i}\right)\left(q^{2} ; q\right)_{\infty}} .
\end{aligned}
$$

Proof By the definition of $q$-integral (1.7), we get

$$
\begin{aligned}
& \left|\int_{c}^{d}{ }_{r+1} \phi_{r}\left(\begin{array}{c}
a_{1}, a_{2}, \ldots, a_{r+1} ; q, z \\
b_{1}, b_{2}, \ldots, b_{r}
\end{array}\right) d_{q} z\right| \\
& =\left|\int_{0}^{d}{ }_{r+1} \phi_{r}\left(\begin{array}{c}
a_{1}, a_{2}, \ldots, a_{r+1} \\
b_{1}, b_{2}, \ldots, b_{r}
\end{array} ; q, z\right) d_{q} z-\int_{0}^{c}{ }_{r+1} \phi_{r}\left(\begin{array}{c}
a_{1}, a_{2}, \ldots, a_{r+1} \\
b_{1}, b_{2}, \ldots, b_{r}
\end{array} ; q, z\right) d_{q} z\right|
\end{aligned}
$$

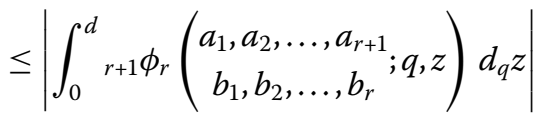

$$
\begin{aligned}
& +\left|\int_{0}^{c}{ }_{r+1} \phi_{r}\left(\begin{array}{c}
a_{1}, a_{2}, \ldots, a_{r+1} ; q, z \\
b_{1}, b_{2}, \ldots, b_{r}
\end{array}\right) d_{q} z\right| \\
& \leq \frac{|d|}{\left(1-|d| \prod_{i=1}^{r+1} M_{i}\right)\left(q^{2} ; q\right)_{\infty}}+\frac{|c|}{\left(1-|c| \prod_{i=1}^{r+1} M_{i}\right)\left(q^{2} ; q\right)_{\infty}} \\
& =\frac{|d|+|c|-2|d c| \prod_{i=1}^{r+1} M_{i}}{\left(1-|d| \prod_{i=1}^{r+1} M_{i}\right)\left(1-|c| \prod_{i=1}^{r+1} M_{i}\right)\left(q^{2} ; q\right)_{\infty}} \text {. }
\end{aligned}
$$

Thus, the inequality (2.6) holds.

\section{Some applications of the inequality}

In this section, we use the inequality obtained in this paper to give a sufficient condition for convergence of a $q$-series. Convergence is an important problem in the study of a $q$-series. There are some results about it. For example, Ito used an inequality technique to give a sufficient condition for the convergence of a special $q$-series called the Jackson integral $[15]$.

Theorem 3.1 Suppose $a_{i}, b_{i}$ are any real numbers such that $b_{i}<1$ with $i=1,2, \ldots, r$. Let $\left\{c_{n}\right\}$ be any number series. If

$$
\lim _{n \rightarrow \infty}\left|\frac{c_{n+1}}{c_{n}}\right|=p<\frac{1}{q}
$$

then the q-series

$$
\sum_{n=0}^{\infty} c_{n} \int_{0}^{q^{n}}{ }_{r+1} \phi_{r}\left(\begin{array}{c}
a_{1}, a_{2}, \ldots, a_{r+1} \\
b_{1}, b_{2}, \ldots, b_{r}
\end{array} ;, z\right) d_{q} z
$$

converges absolutely. 
Proof Since

$$
\lim _{n \rightarrow \infty} q^{n}=0
$$

there exists an integer $N_{0}$ such that, when $n>N_{0}$,

$$
q^{n}<\left(\prod_{i=1}^{r+1} M_{i}\right)^{-1}
$$

where $b_{r+1}=0, M_{i}=\max \left\{1, \frac{\left|1-a_{i}\right|}{1-b_{i}}\right\}$ for $i=1,2, \ldots, r+1$.

When $n>N_{0}$, letting $t=q^{n}$ in (1.1) gives

$$
\left|\int_{0}^{q^{n}}{ }^{n+1} \phi_{r}\left(\begin{array}{c}
a_{1}, a_{2}, \ldots, a_{r+1} \\
b_{1}, b_{2}, \ldots, b_{r}
\end{array} ;, z\right) d_{q} z\right| \leq \frac{q^{n}}{\left(1-q^{n} \prod_{i=1}^{r+1} M_{i}\right)\left(q^{2} ; q\right)_{\infty}} .
$$

Multiplying both sides of (3.4) by $\left|c_{n}\right|$ one gets

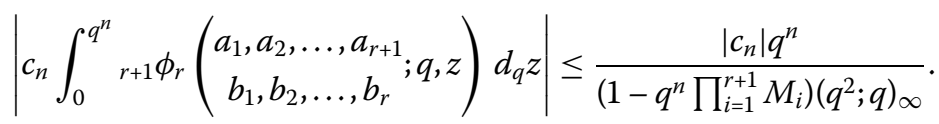

The ratio test shows that the series

$$
\sum_{n=0}^{\infty} \frac{c_{n} q^{n}}{\left(1-q^{n} \prod_{i=1}^{r+1} M_{i}\right)\left(q^{2} ; q\right)_{\infty}}
$$

is absolutely convergent. From (3.5), it is sufficient to establish that (3.1) is absolutely convergent.

Corollary 3.2 Suppose $a_{i}, b_{i}$ are any real numbers such that $b_{i}<1$ with $i=1,2, \ldots, r$. Then the q-integral

$$
\int_{0}^{1} d_{q} t \int_{0}^{t}{ }_{r+1} \phi_{r}\left(\begin{array}{c}
a_{1}, a_{2}, \ldots, a_{r+1} \\
b_{1}, b_{2}, \ldots, b_{r}
\end{array} ;, z\right) d_{q} z
$$

is absolutely convergent. Here

$$
\begin{aligned}
& \int_{0}^{1} d_{q} t \int_{0}^{t}{ }_{r+1} \phi_{r}\left(\begin{array}{c}
\left.a_{1}, a_{2}, \ldots, a_{r+1} ; q, z\right) d_{q} z \\
b_{1}, b_{2}, \ldots, b_{r}
\end{array}\right) \\
& \quad=\int_{0}^{1}\left[\int_{0}^{t}{ }^{r+1} \phi_{r}\left(\begin{array}{c}
a_{1}, a_{2}, \ldots, a_{r+1} ; q, z \\
b_{1}, b_{2}, \ldots, b_{r}
\end{array}\right) d_{q} z\right] d_{q} t .
\end{aligned}
$$

Proof By the definition of $q$-integral (1.7), we get

$$
\begin{aligned}
& \int_{0}^{1} d_{q} t \int_{0}^{t}{ }_{r+1} \phi_{r}\left(\begin{array}{c}
\left.a_{1}, a_{2}, \ldots, a_{r+1} ; q, z\right) d_{q} z \\
b_{1}, b_{2}, \ldots, b_{r}
\end{array}\right) \\
& \quad=(1-q) \sum_{n=0}^{\infty} q^{n} \int_{0}^{q^{n}}{ }^{r+1} \phi_{r}\left(\begin{array}{c}
\left.a_{1}, a_{2}, \ldots, a_{r+1} ; q, z\right) d_{q} z . \\
b_{1}, b_{2}, \ldots, b_{r}
\end{array}\right)
\end{aligned}
$$


Since

$$
\lim _{n \rightarrow \infty}\left|\frac{q_{n+1}}{q_{n}}\right|=q<\frac{1}{q}
$$

from the theorem, we know that (3.6) is absolutely convergent.

\section{Competing interests}

The author declares that he has no competing interests.

\section{Acknowledgements}

The author was supported by the National Natural Science Foundation (grant 11271057) of China.

Received: 20 April 2014 Accepted: 30 June 2014 Published: 22 Jul 2014

\section{References}

1. Anderson, GD, Barnard, RW, Vamanamurthy, KC, Vuorinen, M: Inequalities for zero-balanced hypergeometric functions. Trans. Am. Math. Soc. 347(5), 1713-1723 (1995)

2. Aral, A, Gupta, V, Agarwal, RP: Applications of q-Calculus in Operator Theory Applications. Springer, Berlin (2013)

3. Cao, J: Notes on Askey-Roy integral and certain generating functions for q-polynomials. J. Math. Anal. Appl. 409(1), 435-445 (2014)

4. Ernst, T: The history of $q$-calculus and a new method. Licentiate Thesis, U.U.D. M Report (2000)

5. Giordano, C, Laforgia, A, Pečarić, J: Supplements to known inequalities for some special functions. J. Math. Anal. Appl. 200, 34-41 (1996)

6. Giordano, C, Laforgia, A, Pečarić, J: Unified treatment of Gautschi-Kershaw type inequalities for the gamma function. J. Comput. Appl. Math. 99, 167-175 (1998)

7. Giordano, C, Laforgia, A: Inequalities and monotonicity properties for the gamma function. J. Comput. Appl. Math. $133,387-396(2001)$

8. Giordano, C, Laforgia, A: On the Bernstein-type inequalities for ultraspherical polynomials. J. Comput. Appl. Math. 153, 243-284 (2003)

9. Örkcü, M: Approximation properties of bivariate extension of q-Szász-Mirakjan-Kantorovich operators. J. Inequal. Appl. 2013, 324 (2013)

10. Tariboon, J, Ntouyas, SK: Quantum integral inequalities on finite intervals. J. Inequal. Appl. 2014, 121 (2014)

11. Andrews, GE: The Theory of Partitions. Encyclopedia of Mathematics and Applications, vol. 2. Addison-Wesley, Reading (1976)

12. Gasper, G, Rahman, M: Basic Hypergeometric Series. Cambridge University Press, Cambridge (1990)

13. Jackson, FH: On q-definite integrals. Q. J. Pure Appl. Math. 50, 101-112 (1910)

14. Wang, M: An inequality for ${ }_{r+1} \phi_{r}$ and its applications. J. Math. Inequal. 1, 339-345 (2007)

15. Ito, M: Convergence and asymptotic behavior of Jackson integrals associated with irreducible reduced root systems. J. Approx. Theory 124, 154-180 (2003)

10.1186/1029-242X-2014-268

Cite this article as: Wang: An inequality for $q$-integral and its applications. Journal of Inequalities and Applications 2014, 2014:268

\section{Submit your manuscript to a SpringerOpen ${ }^{\ominus}$ journal and benefit from:}

- Convenient online submission

- Rigorous peer review

- Immediate publication on acceptance

- Open access: articles freely available online

- High visibility within the field

- Retaining the copyright to your article 